\title{
3 Dimensional Microscopy, Serial Block Face SEM
}

Joel Mancuso

Gatan Inc., Pleasanton, CA

An essential biological question is "how is the three-dimensional geometry of cellular structures organized?" Life science researchers have two options for pursuing this question at the transmission electron microscope (TEM) level, tomography, and serial section imaging. Although tomography has excellent resolution, the field of view is limited and it is impractical for large volume acquisitions. Serial sectioning is used for obtaining large volume three-dimensional 3D electron microscopic data, but the technique is a highly skilled time consuming process prone to human error. Serial block-face scanning electron microscopy (SBFSEM) is a new automated technique in which a scanning electron microscope is used to obtain serial images. Bridging the gap between ultrahigh resolution tomography and fluorescence microscopy, SBFSEM provides a streamlined and automated 3D data acquisition process.

A microtome equipped with a diamond knife is mounted inside the chamber of the SEM and shaves the surface of the sample in between imaging. Images are collected using a back scatter (BS) electron detector which provides a classic TEM-like image. The SBFSEM imaging process is completely automated allowing for large volume acquisition in a timely manner without the risk of losing sections. SBFSEM was previously hindered by resolution barriers; recently we have demonstrated resolution improvements with the ability to resolve microtubules, and synaptic vesicles. This improvement is due to new specimen preparation techniques involving enhanced staining. This staining mechanism not only improves the signal to noise ration, but allows the biological sample embedded in epoxy resin to be imaged in high vacuum conditions.

With resolution improvements, 3 View also has enabled multiposition acquisition. This allows large volume high resolution data. In addition, the new staining strategy also allows imaging with lower accelerating voltages reducing the interaction volume. Matching the interaction volume to the slice thickness is import to reduce any signal contribution from a volume not removed by the following cut. We will demonstrate optimal imaging parameters by correlating monte carlo simulation data along with experimental data to choose the appropriate $\mathrm{keV}$ and slice thickness.

References:

[1] Denk W, Horstmann H. (2004). Serial block-face scanning electron microscopy to reconstruct threedimensional tissue nanostructure. PLoS Biol. 2/e329:1900-1909; 329.

[2] West JB, Fu Z, Deerinck TJ, Mackey MR, Obayashi JT, Ellisman MH. Structure-function studies of blood and air capillaries in chicken lung using 3D electron microscopy. Respir Physiol Neurobiol. 2010 Feb 28;170(2):202-209. Epub 2009 Dec 28.

Figure A. Raw data of Rat cerebellum. Original image size is 4096x4096 with a field of view of $25 \mu \mathrm{m}$. Figure B. High resolution image of highlighted area in figure A, showing synaptic vesicles.

Figure C. Individual neurons segmented with Imaris software.

Figure D. Complet volume visualized with the 3D visualization plugin for DigitalMicrograph. 

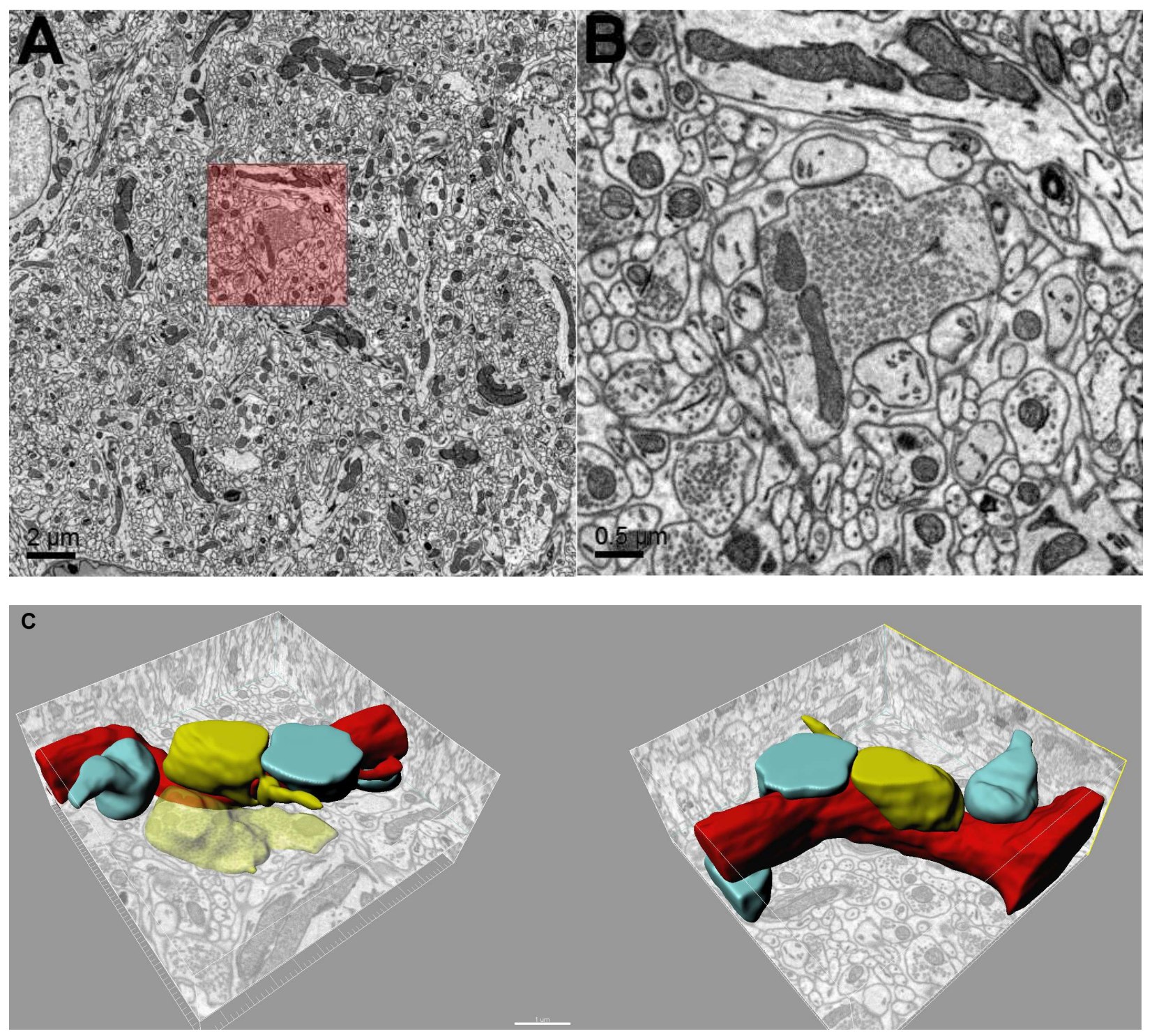

\section{D}

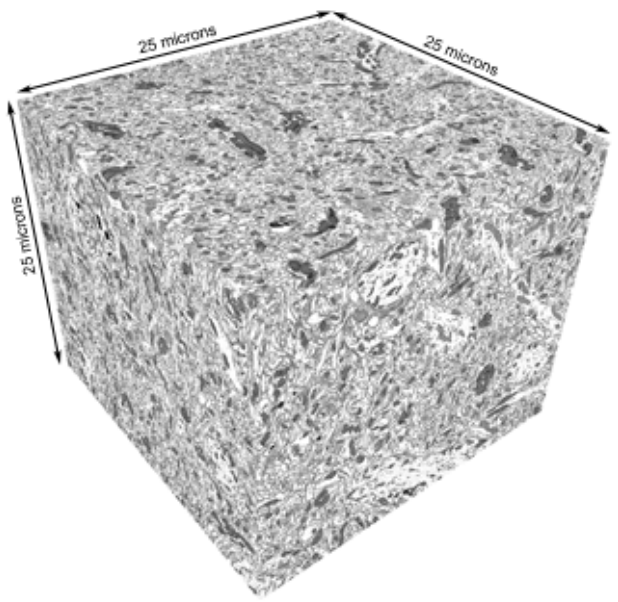

